\title{
Insulin-Like Growth Factor-1 Receptor Targeted Fluorescent Imaging for Gallbladder Cancer in Orthotopic Mouse Models
}

\author{
Jung $\mathrm{Ha}$ Choi and Jeong Youp Park \\ Division of Gastroenterology, Department of Internal Medicine and Institute of Gastroenterology, Severance Hospital, Yonsei University \\ College of Medicine, Seoul, Korea
}

\author{
Article Info \\ Received April 10, 2021 \\ Revised June 22, 2021 \\ Accepted July 13, 2021 \\ Published online September 1, 2021

\section{Corresponding Author} \\ Jeong Youp Park \\ ORCID https://orcid.org/0000-0003-0110-8606 \\ E-mail sensass@yuhs.ac
}

\begin{abstract}
Background/Aims: Gallbladder cancer is fatal, but fluorescent imaging technology can facilitate timely diagnosis and improve patient outcomes. Fluorophore-conjugated insulin-like growth factor-1 receptor (IGF-1R) targeted antibodies were used to visualize gallbladder cancer in orthotopic tumor mouse models.

Methods: Western blotting, flow cytometric analysis, and confocal microscopy detected the expression of IGF-1R in SNU-308, SNU-478, and SNU-1196 bile duct cancer cells. In vivo imaging of SNU-478 and SNU-1196 subcutaneous tumors and orthotopic gallbladder tumor models of SNU-478 were performed after injection with DyLight 650-conjugated IGF-1R antibody.

Results: Western blotting and flow cytometric analysis showed that IGF-1R was expressed in bile duct cancer cells, and confocal microscopy demonstrated that IGF-1R antibody was able to bind to IGF-1R on the cell membrane. Fluorescent IGF-1R antibody injected into the mouse tail vein made subcutaneous tumors and orthotopic tumors become fluorescent. The intensity of fluorescence from the tumor was stronger than that from surrounding normal tissues. Histochemical examination confirmed that the tumor was located inside the gallbladder and adjacent liver parenchyma of mice.

Conclusions: Our study showed that a fluorescent IGF-1R-targeted antibody could help detect gallbladder tumors. Tumor-specific imaging technology can be applied to endoscopy, laparoscopy, and robotic surgery for better management of gallbladder cancer. (Gut Liver 2022;16:606612)
\end{abstract}

Key Words: Gallbladder neoplasms; Insulin-like growth factor-1 receptor; Imaging

\section{INTRODUCTION}

Gallbladder cancer is a potentially fatal cancer responsible for $1.7 \%$ of all cancer mortality. ${ }^{1}$ Because it is difficult to distinguish gallbladder cancer from benign gallbladder disease, many cases are diagnosed incidentally after cholecystectomy. ${ }^{2}$ Incidental gallbladder cancer represents between $1.6 \%$ and $50 \%$ of all gallbladder cancers. Incidental gallbladder cancer detection raises several medical problems. First, a second intervention is needed to ensure complete surgical resection and tumor staging. In addition, unexpectedly resecting gallbladder cancer may cause cancer cell dissemination. ${ }^{3}$ This is one of the many reasons that gallbladder cancer is so deadly; thus, it is important to develop a better diagnostic method for gallbladder cancer to ensure timely treatment.

Fluorescent imaging technology may help to diagnose gallbladder cancer. Previous studies reported that fluorescent imaging could improve tumor visualization and surgical outcomes of colon cancer using fluorescent carcinoembryonic antigen antibody. Type I insulin-like growth factor-1 receptor (IGF-1R), a transmembrane tyrosine kinase expressed in pancreatic and colon cancers, can be a potential target for fluorescent imaging. ${ }^{5,6}$ Gallbladder cancer might be another cancer for which IGF-1R-targeted fluorescent imaging is useful.

In this study, we sought to demonstrate that fluorophore-conjugated IGF-1R-targeted antibodies could be 
used to visualize gallbladder cancer in orthotopic tumor mouse models.

\section{MATERIALS AND METHODS}

\section{Gallbladder cancer cell lines}

The human bile duct cancer cell lines SNU-308, SNU478, and SNU-1196 (Korean Cell Line Bank, Seoul, Korea) were maintained in RPMI 1640 and Minimum Essential Medium with Earle's Balanced Salts medium supplemented with $10 \%$ fetal bovine serum (Gibco, Carlsbad, CA, USA), and antibiotic-antimycotic solution (Gibco). All cells were cultured at $37^{\circ} \mathrm{C}$ in a $5 \% \mathrm{CO}_{2}$ incubator.

\section{Mice}

Balb/c nude mice (Central Lab. Animal, Inc., Seoul, Korea), 4 to 5 weeks old, were used in the study. Mice were kept in a barrier facility under high efficiency particulate air filtration and fed with an autoclaved laboratory rodent diet. All mouse surgical procedures and imaging were performed after anesthetization by intramuscular injection of $60 \%$ tiletamine and zolazepam, and $40 \%$ xylazine $\mathrm{HCl}$ $(0.02 \mathrm{~mL})$. Animals received antibiotics immediately prior to surgery and once a day over the next 3 days. The maximum tumor size allowed was $2 \mathrm{~cm}$. The condition of the animals was monitored every day. $\mathrm{CO}_{2}$ inhalation was used for euthanasia. This study was approved by and performed in accordance with the guidelines of the Yonsei University Health System Institutional Animal Care and Use Committee (IACUC number: 2020-0015).

\section{Antibody-dye conjugation}

Mouse monoclonal antibodies to IGF-1R $\alpha$ (clone 2431; Thermo Scientific, Rockford, IL, USA) were conjugated with DyLight 650 dyes (Thermo Scientific) after removing bovine serum albumin with Pierce ${ }^{\mathrm{TM}}$ Antibody Clean-up Kit (Thermo Scientific) per manufacturer specifications. ${ }^{5,6}$

\section{Western blotting}

Cell lysates were extracted in lysis buffer containing 70 $\mathrm{mM} \beta$-glycerophosphate, $0.6 \mathrm{mM}$ sodium orthovanadate, $2 \mathrm{mM} \mathrm{MgCl}_{2}, 1 \mathrm{mM}$ ethylene glycol tetraacetic acid, $1 \mathrm{mM}$ DTT (Invitrogen, Grand Island, NY, USA), 0.5\% TritonX100, $0.2 \mathrm{mM}$ phenylmethylsulfonyl fluoride, and $1 \%$ protease inhibitor cocktail (Sigma-Aldrich, St. Louis, MO, USA). Lysates were separated via sodium dodecyl sulfatepolyacrylamide gel electrophoresis and transferred to polyvinylidene fluoride membranes (Millipore, Billerica, MA, USA). The membranes were blocked in $5 \%(\mathrm{w} / \mathrm{v})$ nonfat dry milk and probed with anti-IGF-1R $\alpha$ (N-20; Santa
Cruz, Dallas, TX, USA) at dilution of 1:200. The immunoreactive proteins were visualized using the SuperSignal West Pico Chemiluminescent Substrate (Thermo Scientific).

\section{Flow cytometric analysis and confocal microscope imaging}

A total of $2 \times 10^{6}$ cells were washed with phosphatebuffered saline and fixed with $4 \%$ paraformaldehyde for 15 minutes at room temperature (in the dark). The cells were washed and blocked with $1 \%$ bovine serum albumin, for 1 hour at room temperature. After incubation, they were washed with PBS and cells were incubated with IGF-1R $\alpha$ (clone 24-31, $5 \mathrm{nM}$; Thermo Scientific) at $5 \mathrm{nM}$ for 3 hours at room temperature. They were then incubated with Alexa fluor 488-conjugated polyclonal goat anti-mouse secondary antibody (Jackson ImmunoResearch Laboratories, Inc., West Grove, PA, USA) for 1 hour at room temperature. After washing with PBS, cells were detected with BD FACS LSRII SORP system (Becton Dickinson Company, Franklin Lakes, NJ, USA) and confocal microscope LSM 700 (Carl Zeiss, Oberkochen, Germany). Images acquired by confocal microscope were merged with Adobe Photoshop 6.0 .

\section{Subcutaneous and orthotopic tumor mouse models}

Cell lines for subcutaneous and orthotopic tumor models were chosen by the success rate of tumor engraftment and time taken to make them. To make subcutaneous tumor models, SNU-478 and SNU-1196 $\left(0.2 \mathrm{~mL}, 2 \times 10^{6}\right)$ were injected subcutaneously into the flanks of $\mathrm{Balb} / \mathrm{c}$ nude mice. When the size of subcutaneous tumors reached between 10 and $20 \mathrm{~mm}$ in diameter, imaging was performed. To create the orthotopic tumor mouse models, $2 \times 10^{6}$ cells of SNU-478 were mixed with Matrigel (Corning, Glendale, AZ, USA). The gallbladder was quickly exposed, and then $40 \mu \mathrm{L}$ of cell suspension mixed with Matrigel was injected into the gallbladder slowly using a 29-gauge insulin syringe (Becton Dickinson Company). After waiting for approximately 30 seconds, the gallbladder and liver lobes were replaced and the abdominal wall was closed using Vicryl 6/0. The physical condition of the mice was monitored every day in the first week.

\section{Immunohistochemistry}

The formalin-fixed, paraffin-embedded tissue samples were sectioned into serial $4-\mathrm{mm}$ slices and placed on microscope slides. After deparaffinization in xylene and rehydration in alcohol, tissue sections underwent antigen retrieval for 30 minutes in Tris-EDTA buffer titrated to $\mathrm{pH}$ 9.0 at $97^{\circ} \mathrm{C}$ and cooling with tap water. Nonspecific anti- 
gen reactions were blocked by 1 hour with M.O.M. mouse IgG blocking buffer. The slides were then incubated with the anti-IGF-1R $\alpha$ (clone 24-31; Santa Cruz, Dallas, TX, USA) at dilution of $1: 10$ for 12 to 16 hours at $4^{\circ} \mathrm{C}$, rinsed, and incubated in $3 \%$ hydrogen peroxide $\left(\mathrm{H}_{2} \mathrm{O}_{2}\right)$ for 10 minutes to block endogenous peroxidase activity. After rinsing slides with TBST, a DAKO Real EnVision detection system peroxidase/DAB (DAKO, Glostrup, Denmark) was used, following the manufacturer's instructions. The slides were incubated with $\mathrm{ABC}$ reagent (Vector Laboratories, Inc., Burlingame, CA, USA) for 30 minutes at room temperature and DAB (DAKO) for 3 minutes. The slides were counterstained with hematoxylin for 2 minutes and washed with tap water. Finally, the samples were fixed via immersion in an ethanol dilution series, final immersion in xylene, and sealed with permanent mounting medium (Sigma-Aldrich).

\section{Imaging}

Subcutaneous and orthotopic tumor mouse models were injected with IGF-1R antibody (conjugated with DyLight 650) via the tail vein. Then, imaging was performed using an IVIS ${ }^{\infty}$ Spectrum In Vivo Imaging System (Perkin Elmer, Inc., Waltham, MA, USA). For subcutaneous tumor models, imaging was done on days 1, 2, and 3 after injection under anesthesia. For orthotopic tumor models, imaging was done after euthanasia, opening the abdominal wall, and exposing the liver and gallbladder.

After in vivo imaging of orthotopic tumor models, the tumor and liver were removed from the mice, and ex vivo imaging was done. Hematoxylin and eosin staining of resected orthotopic tumors was performed and samples were observed under a light microscope.

\section{RESULTS}

\section{Expression of IGF-1R in bile duct cancer cells in vitro}

In vitro experiments confirmed that IGF-1R was expressed in bile duct cancer cells, and fluorescent IGF-1R antibody was able to bind to IGF-1R on the membrane of cancer cells. Western blotting showed expression of IGF1R in SNU-308, SNU-478, and SNU-1196 (Fig. 1A). Flow cytometry revealed expression of IGF-1R in $83.9 \%, 85.9 \%$, and $95.1 \%$ of SNU-308, SNU-478 and SNU-1198 cells (Fig. 1B). Confocal microscopy showed fluorescence foci along the membranes of the cells showing fluorescent IGF-1R antibody was bound to IGF-1R on the cell membrane of bile duct cancer cells (Fig. 1C).

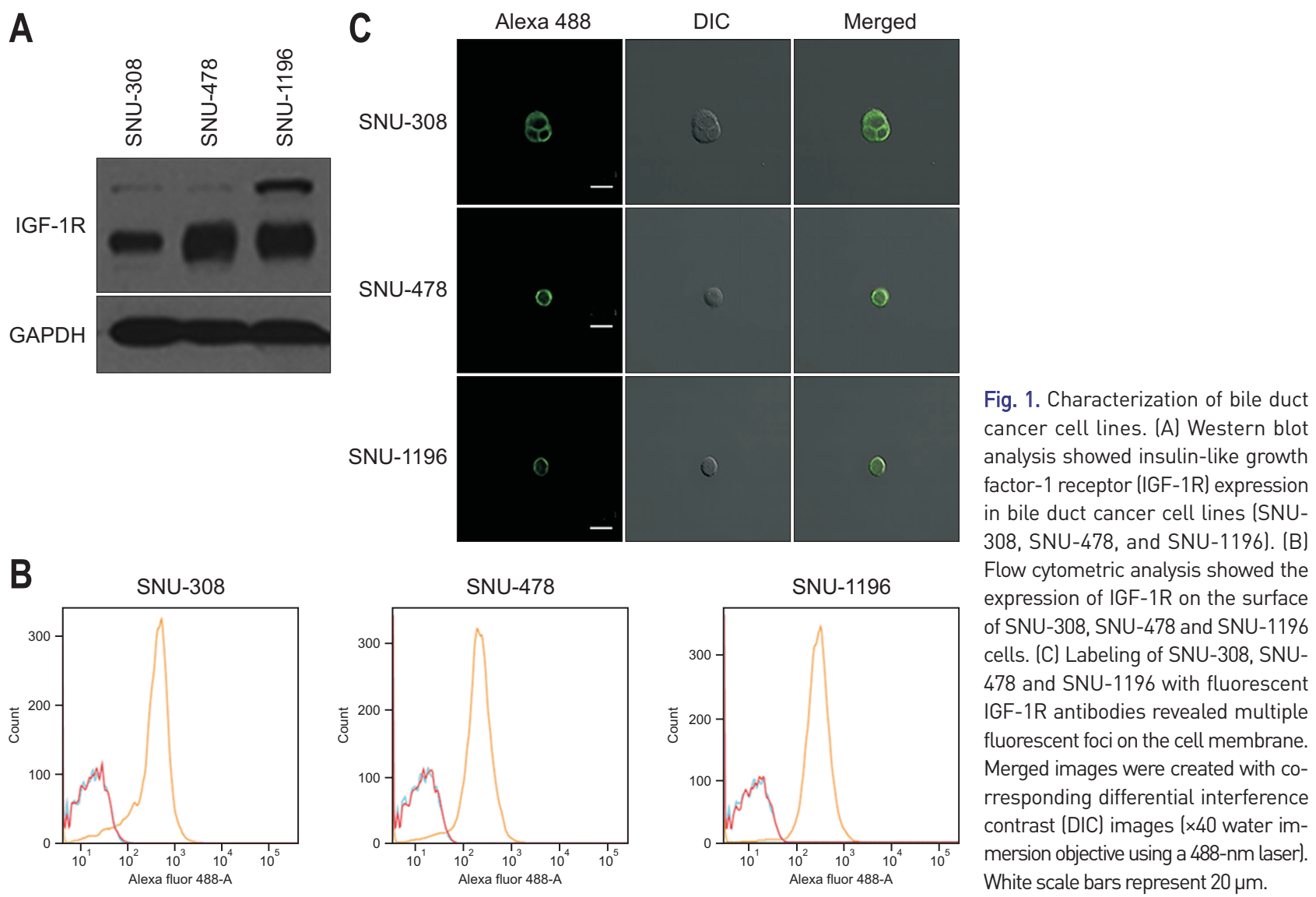




\section{Imaging subcutaneous tumors in nude mice with fluorescent IGF-1R antibodies}

Subcutaneous tumor models were created with SNU478 and SNU-1196, with tumors grown for 4 to 5 weeks after injecting tumor cells in the flanks of mice. After injecting DyLight 650-conjugated IGF-1R antibody was injected into the tail vein of mice, the mice were imaged via IVIS ${ }^{\oplus}$. Twenty-four hours after injection, a strong fluorescent signal was detected in the SNU-478 and SNU-1196 tumors. The intensity of fluorescence from the tumor was stronger than background. As time went on, the intensity of the fluorescent signal decreased, but it was maintained for up to 72 hours (Fig. 2A). Fluorescence was observed in all the mice with tumor.

Immunohistochemical analysis with IGF-1R antibody on resected subcutaneous tumor from mouse models not injected with fluorescent antibody confirmed the expression of IGF-1R in SNU-478 and SNU-1196 tumors (Fig. 2B).

\section{Imaging orthotopic tumors in nude mice with fluorescent IGF-1R antibodies}

Five weeks after injecting cancer cells mixed with

A
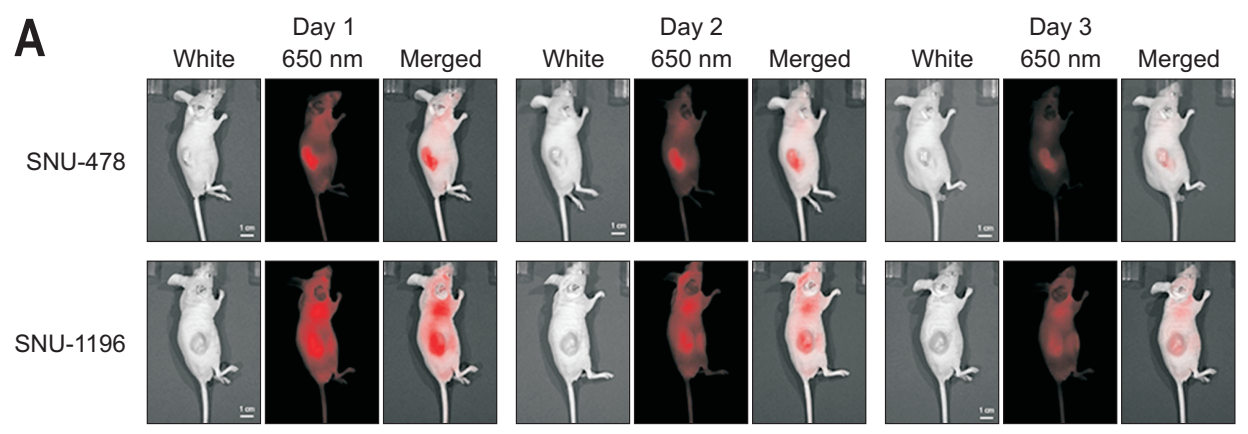

B

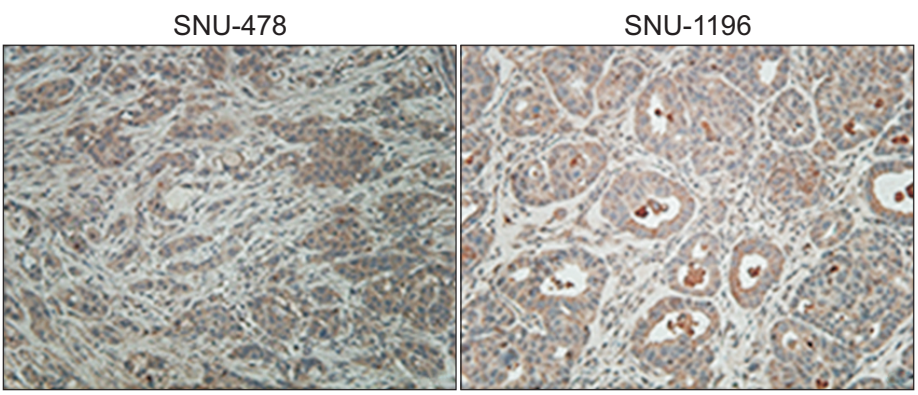

Fig. 2. Imaging of subcutaneously transplanted gallbladder tumors in vivo with a fluorescent insulin-like growth factor-1 receptor (IGF-1R) antibody. (A) Representative images are shown. The mouse was imaged under both white light and fluorescence illumination from day 1 to 3 after injection of the fluorescent IGF-1R antibody. The intensity of the fluorescence signal from subcutaneous SNU-478 and SNU-1196 tumors was stronger than background. As time went on, the intensity of the fluorescent signal decreased, but it was maintained for up to 72 hours. White scale bars represent $1 \mathrm{~cm}$. (B) Immunostaining for IGF-1R on SNU-478 and SNU-1196 tumor tissues (×200).
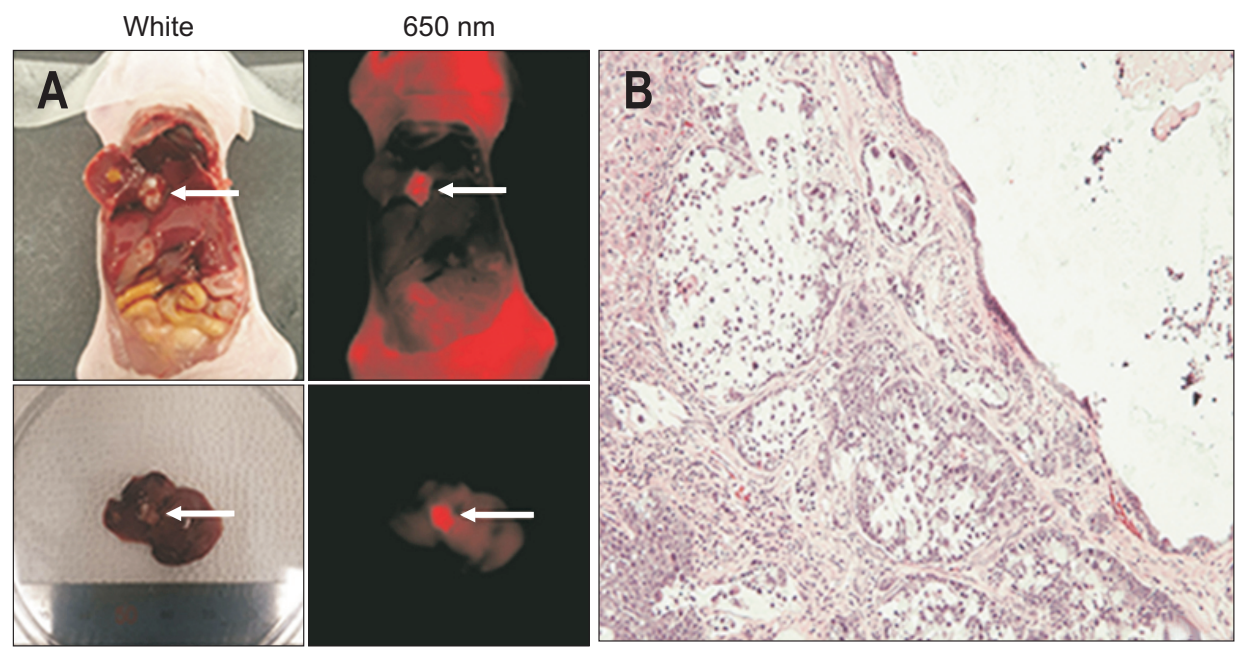

Fig. 3. Imaging of an orthotopically transplanted SNU-478 gallbladder tumor in vivo with fluorescent insulin-like growth factor-1 receptor antibody. (A) Fluorescence signals from gallbladder tumors orthotopically transplanted into the gallbladder were detected. Other than from the tumor, fluorescence signals were detected from the bowel and skin. Fluorescence was also detected in the resected tumor, but not in normal liver. Arrows indicate the gallbladder tumor. (B) Hematoxylin and eosin staining $(\times 200)$. 
Matrigel into the gallbladder, fluorescent IGF-1R antibody was injected into the tails of mice. Twenty-four hours after injecting the antibody, mice were sacrificed and in vivo imaging was performed. After opening the abdomen, tumors were noted in the gallbladders of mice. Fluorescent imaging detected strong signals from the tumor. The intensity of fluorescence was stronger than surrounding normal liver tissues. Intermediate intensity of fluorescent signal was detected from the bowel and skin (Fig. 3A). Ex vivo imaging also confirmed stronger fluorescence from the tumor than the liver (Fig. 3A). No fluorescence was observed from the gallbladder of mice in which tumor was not made. Histochemical examination confirmed that tumor was located inside the gallbladder and adjacent liver parenchyma of mice (Fig. 3B).

\section{DISCUSSION}

Based on many preclinical studies that showed the potential of fluorescent imaging technology, approximately 40 clinical trials for ovarian, renal, lung, head and neck, pancreatic, colorectal, and breast cancer have been conducted using fluorescent technology. ${ }^{7}$ It can help to differentiate malignant tumor from normal tissues without pathologic examination. In addition, it could help achieve complete oncological resection by assessing tumor margins and locoregional invasion. ${ }^{4,8}$ Thus, fluorescent imaging might be useful in cases of gallbladder cancer. In this study, we showed that IGF-1R-targeted fluorescent antibody caused gallbladder tumor to fluoresce, enabling visualization in animal models. Previous studies demonstrated that the same antibody could be used to image pancreatic and colon cancer. ${ }^{5,6}$

Fluorescent imaging in subcutaneous and orthotopic gallbladder tumor animal models showed that IGF-1R antibody could be used to make tumor more fluorescent than surrounding normal tissues. This suggests that fluorescent imaging could help to avoid incidental resection of gallbladder cancer by facilitating diagnosis before and during surgery. In addition, the size of detected tumors was approximately $6 \mathrm{~mm}$, suggesting that even very tiny occult tumors, which might be missed otherwise, could be detected. ${ }^{6}$ In terms of clinical benefit, oncological resection can be achieved and the risk of cancer dissemination can be minimized.

IGF-1R is involved in cell proliferation, apoptosis, angiogenesis, and tumor invasion. ${ }^{9,10}$ It has been considered a potential biomarker of various cancers, ${ }^{9}$ and as a target for several imaging methods in pancreatic, colon, sarcoma and breast cancer. ${ }^{5,6,11,12}$ Expression of IGF-1R in bile duct cancer was also previously reported. ${ }^{13-15}$ There is a report that IGF-1, IGF-II, and IGF-1R were expressed in $45 \%$, 25\%, and $95 \%$ of gallbladder cancer. ${ }^{16}$ Western blot in our study confirmed that bile duct cancer cells did express IGF-1R. FACS and confocal microscopy showed that fluorophoreconjugated IGF-1R antibody was able to bind to IGF-1R on the membrane of cancer cells specifically. In vitro results were concordant with imaging results.

In this study we used fluorophore dye with $650 \mathrm{~nm}$ wavelength. Visible, near infrared, and infrared wavelength of light can be used for fluorescent cancer imaging. Each of them has advantage and disadvantage. Longer wavelength dyes tend to penetrate more deeply. Shorter wavelength dyes are more photostable. ${ }^{17}$ Therefore, each dye should be tested in various clinical settings. Since the gallbladder is near the liver, we used a fluorophore dye tested in liver metastasis model from previous study. ${ }^{6}$ To further prove clinical utility of fluorescent imaging of gallbladder cancer, we are currently testing $800 \mathrm{~nm}$ fluorophore-conjugated antibody which can be detected by laparoscopic and robotic surgery system. ${ }^{18}$

There is a need for suitable gallbladder tumor animal models to develop new treatment options for this disease. ${ }^{19}$ A few studies using orthotopic gallbladder tumor models have been used to prove the effectiveness of different treatment methods. ${ }^{20,21}$ Our study showed that gallbladder orthotopic tumor models could be also useful for developing diagnostic methods, specifically imaging studies to differentiate malignant gallbladder diseases from benign gallbladder diseases and surrounding normal tissue. Even though technique of engrafting tumor can be challenging and cost more money, orthotopic tumor models can be useful for biomedical imaging research because it mimics clinical situations better than traditionally used subcutaneous tumor models. ${ }^{22}$ Not only fluorescent agents, but also radionuclide could be conjugated to IGF-1R antibody for single photon emission computed tomography and positron emission tomography. ${ }^{11,23,24}$ Orthotopic gallbladder tumor models can be utilized for researches in such imaging technology, which can help to manage gallbladder cancer better.

In conclusion, our study showed that IGF-1R-targeted antibody conjugated with fluorophore could be used to detect gallbladder tumor through fluorescence. Tumorspecific imaging technology could be applied to endoscopy, laparoscopy, robotic surgery, and various imaging equipment for better management of gallbladder cancer. 


\section{CONFLICTS OF INTEREST}

No potential conflict of interest relevant to this article was reported.

\section{ACKNOWLEDGEMENTS}

This study was supported by grant from the Korea Research Foundation under the basic research promotion fund of the Ministry of Education and Health Resources Development of Korea (number: 2019-31-0725) and by a faculty research grant from Yonsei University College of Medicine (number: 6-2018-0071).

\section{AUTHOR CONTRIBUTIONS}

Conceptualization: J.Y.P. Data curation: J.H.C., J.Y.P. Formal analysis: J.H.C., J.Y.P. Funding acquisition: J.Y.P. Methodology: J.H.C., J.Y.P. Project administration: J.Y.P. Visualization: J.H.C. Writing - original draft: J.H.C. Writing - review \& editing: J.Y.P. Approval of final manuscript: all authors.

\section{ORCID}

Jung Ha Choi https://orcid.org/0000-0002-0065-3699 Jeong Youp Park https://orcid.org/0000-0003-0110-8606

\section{REFERENCES}

1. Rawla P, Sunkara T, Thandra KC, Barsouk A. Epidemiology of gallbladder cancer. Clin Exp Hepatol 2019;5:93-102.

2. Hickman L, Contreras C. Gallbladder cancer: diagnosis, surgical management, and adjuvant therapies. Surg Clin North Am 2019;99:337-355.

3. Kellil T, Chaouch MA, Aloui E, et al. Incidence and preoperative predictor factors of gallbladder cancer before laparoscopic cholecystectomy: a systematic review. J Gastrointest Cancer 2021;52:68-72.

4. Hiroshima Y, Maawy A, Metildi CA, et al. Successful fluorescence-guided surgery on human colon cancer patientderived orthotopic xenograft mouse models using a fluorophore-conjugated anti-CEA antibody and a portable imaging system. J Laparoendosc Adv Surg Tech A 2014;24:241-247.

5. Park JY, Lee JY, Zhang Y, Hoffman RM, Bouvet M. Targeting the insulin growth factor-1 receptor with fluorescent antibodies enables high resolution imaging of human pan- creatic cancer in orthotopic mouse models. Oncotarget 2016;7:18262-18268.

6. Park JY, Murakami T, Lee JY, Zhang Y, Hoffman RM, Bouvet M. Fluorescent-antibody targeting of insulin-like growth factor-1 receptor visualizes metastatic human colon cancer in orthotopic mouse models. PLoS One 2016;11:e146504.

7. Hernot S, van Manen L, Debie P, Mieog JSD, Vahrmeijer AL. Latest developments in molecular tracers for fluorescence image-guided cancer surgery. Lancet Oncol 2019;20:e354e367.

8. Metildi CA, Kaushal S, Pu M, et al. Fluorescence-guided surgery with a fluorophore-conjugated antibody to carcinoembryonic antigen (CEA), that highlights the tumor, improves surgical resection and increases survival in orthotopic mouse models of human pancreatic cancer. Ann Surg Oncol 2014;21:1405-1411.

9. Pollak MN, Schernhammer ES, Hankinson SE. Insulin-like growth factors and neoplasia. Nat Rev Cancer 2004;4:505518.

10. Maloney EK, McLaughlin JL, Dagdigian NE, et al. An anti-insulin-like growth factor I receptor antibody that is a potent inhibitor of cancer cell proliferation. Cancer Res 2003;63:5073-5083.

11. Heskamp S, van Laarhoven HW, Molkenboer-Kuenen JD, et al. Optimization of IGF-1R SPECT/CT imaging using 111In-labeled $\mathrm{F}\left(\mathrm{ab}^{\prime}\right) 2$ and Fab fragments of the monoclonal antibody R1507. Mol Pharm 2012;9:2314-2321.

12. Zhang H, Zeng X, Li Q, Gaillard-Kelly M, Wagner CR, Yee D. Fluorescent tumour imaging of type I IGF receptor in vivo: comparison of antibody-conjugated quantum dots and small-molecule fluorophore. Br J Cancer 2009;101:71-79.

13. Vaquero J, Lobe C, Tahraoui S, et al. The IGF2/IR/IGF1R pathway in tumor cells and myofibroblasts mediates resistance to EGFR inhibition in cholangiocarcinoma. Clin Cancer Res 2018;24:4282-4296.

14. Suzuki H, Roa JC, Kawamoto T, et al. Expression of insulinlike growth factor I receptor as a biomarker for predicting prognosis in biliary tract cancer patients. Mol Clin Oncol 2015;3:464-470.

15. Xu L, Hausmann M, Dietmaier W, et al. Expression of growth factor receptors and targeting of EGFR in cholangiocarcinoma cell lines. BMC Cancer 2010;10:302.

16. Kornprat P, Rehak P, Rüschoff J, Langner C. Expression of IGF-I, IGF-II, and IGF-IR in gallbladder carcinoma: a systematic analysis including primary and corresponding metastatic tumours. J Clin Pathol 2006;59:202-206.

17. Maawy AA, Hiroshima Y, Kaushal S, Luiken GA, Hoffman RM, Bouvet M. Comparison of a chimeric anti-carcinoembryonic antigen antibody conjugated with visible or near-infrared fluorescent dyes for imaging pancreatic cancer in orthotopic nude mouse models. J Biomed Opt 2013;18:126016. 
18. Ahmad A. Use of indocyanine green (ICG) augmented nearinfrared fluorescence imaging in robotic radical resection of gallbladder adenocarcinomas. Surg Endosc 2020;34:24902494.

19. Egberts JH, Schniewind B, Schafmayer C, et al. Establishment of a novel orthotopic xenograft model of human gallbladder carcinoma. Clin Exp Metastasis 2007;24:141-148.

20. Horiuchi H, Kawamata H, Furihata T, et al. A MEK inhibitor (U0126) markedly inhibits direct liver invasion of orthotopically inoculated human gallbladder cancer cells in nude mice. J Exp Clin Cancer Res 2004;23:599-606.

21. Mita Y, Ajiki T, Kamigaki T, et al. Antitumor effect of gemcitabine on orthotopically inoculated human gallbladder cancer cells in nude mice. Ann Surg Oncol 2007;14:1374-
1380.

22. Lee NP, Chan CM, Tung LN, Wang HK, Law S. Tumor xenograft animal models for esophageal squamous cell carcinoma. J Biomed Sci 2018;25:66.

23. Cornelissen B, McLarty K, Kersemans V, Reilly RM. The level of insulin growth factor-1 receptor expression is directly correlated with the tumor uptake of (111)In-IGF-1(E3R) in vivo and the clonogenic survival of breast cancer cells exposed in vitro to trastuzumab (Herceptin). Nucl Med Biol 2008;35:645-653.

24. Su X, Cheng K, Liu Y, Hu X, Meng S, Cheng Z. PET imaging of insulin-like growth factor type 1 receptor expression with a 64Cu-labeled Affibody molecule. Amino Acids 2015;47:1409-1419. 